\title{
Diagnostic Testing in AKI: Let's Move the Field Forward
}

\author{
Mark A. Perazella, MD
}

Professor of Medicine, Section of Nephrology, Yale University School of Medicine, New Haven, Connecticut.

In this issue of the Journal of Hospital Medicine, Lusica et al. ${ }^{1}$ discuss the utility of urine eosinophils (UEs) in evaluating for acute interstitial nephritis (AIN) in patients with acute kidney injury (AKI), an important and oft-confused concern in medicine. I can't think of a more appropriate topic for the "Things We Do for No Reason" (TWDFNR) series. Numerous tests are ordered in the evaluation of AKI. ${ }^{2}$ Many, such as batteries of serological tests, are unnecessary and add little diagnostic information. Some, such as UEs and fractional excretion of sodium (FENa), provide misinformation. And others, such as contrast-enhanced computed tomography scans, are potentially harmful. ${ }^{2}$ In a previous TWDFNR article, the limitations of FENa in the evaluation of AKI were reviewed. ${ }^{3}$ There are common threads linking the shortcomings of UEs and FENa and even new diagnostic tests. What are the lessons from these studies, and how might clinicians best apply them in their practice?

As reviewed in this issue, UE testing is employed in AKI to evaluate for hospital-acquired AIN. Small initial studies led to widespread use of this test, despite methodological flaws. ${ }^{4}$ A later, definitive study involving 566 patients who had both UEs and kidney biopsies performed within the same week demonstrated that UEs offered no diagnostic value in AKI. ${ }^{5}$ The same pattern occurred in the increased use of FENa to distinguish prerenal azotemia from acute tubular necrosis in AKI patients. ${ }^{3}$ Small studies in highly select patients supported its use for this purpose. ${ }^{6}$ Subsequently, larger studies in more diverse populations noted that FENa was associated with many false positive and negative results, ${ }^{6}$ likely due to more widespread use of this test in disease states such as cirrhosis, congestive heart failure, chronic kidney disease, and diabetes, which were not included in initial studies.

It is apparent that clinicians have been led astray by small, flawed positive studies employed in highly selected populations. These initial positive studies based on excessively large effect size estimates were subsequently shown to be negative in larger studies with more plausible effect sizes. Examples of this error are seen in publications involving prophylactic measures to reduce contrast nephrotoxicity. ${ }^{7}$ Early studies on $\mathrm{N}$-acetylcysteine administration prior to radiocontrast exposure showed positive results. Examination of these studies,

\footnotetext{
*Address for correspondence and reprint requests: Mark A. Perazella, MD, Professor of Medicine, Section of Nephrology, Yale University School of Medicine, 330 Cedar Street, BB 114, New Haven, CT 06520; Telephone: 203-7854184; Fax: 203-785-7068; E-mail: mark.perazella@yale.edu
}

Received: December 9, 2016; Accepted December 10, 2016

2017 Society of Hospital Medicine DOI 10.12788/jhm.2735 however, demonstrates 2 key problems: 1) inclusion of small numbers of patients due to power calculations based on excessively large effect sizes, and 2) use of clinically unimportant endpoints such as serum creatinine changes. ${ }^{7}$ The same issue complicates studies evaluating isotonic sodium bicarbonate vs. normal saline for contrast prophylaxis.

The past 10-plus years have seen a proliferation of studies evaluating the utility of novel biomarkers for early diagnosis and prognosis in AKI. Have we fallen down the same rabbit hole in evaluating these new diagnostic tests for AKI? There is reason for concern if we examine published studies of novel biomarkers in other areas of medicine. To this point, many highly cited novel biomarker studies used for various diagnostic purposes (eg, cancer, infection, cardiovascular disease) employed excessively large effect size estimates for postulated associations that resulted in small, underpowered studies with initially positive results. ${ }^{8}$ Subsequent large studies and meta-analyses reported negative or modestly positive test results when examining these same associations. ${ }^{8}$ But we may be moving in the right direction. An early urine biomarker publication from a small, single center study ${ }^{9}$ revealed overly optimistic results (area under the curve [AUC], 0.998; sensitivity, 100\%; specificity, 98\%) for AKI prediction. Subsequent large, multicenter biomarker studies showed only modest improvement in their discriminative value when compared with traditional clinical models. ${ }^{10}$ These results precluded U.S. Food and Drug Administration (FDA) approval of most novel biomarkers for clinical practice and they were not adopted. In 2014, the FDA approved the point-of-care urinary biomarker TIMP-2/ IGFBP7 (NephroCheck ${ }^{\circledR}$ ) for predicting risk of AKI based on fairly rigorous testing using larger numbers of patients, heterogeneous populations, and important clinical endpoints. ${ }^{11}$ In a 522-patient discovery cohort, this biomarker had an AUC of 0.80 for AKI prediction, which was validated in a 722-patient cohort and subsequently followed by a 420 -patient multicenter cohort study revealing similar test characteristics (AUC, 0.82; sensitivity, 92\%; specificity, 46\%). ${ }^{11}$ A study involving 382 critically ill AKI patients noted that this biomarker had a hazard ratio of $2.16(95 \%$ confidence interval [CI] 1.32 to 3.53) for predicting dialysis requirement or death. ${ }^{11}$ And while this test has yet to find its clinical niche, its operating characteristics are well-studied and likely valid. While predicting AKI earlier does not currently result in effective therapy, it may allow more timely discontinuation or avoidance of potentially nephrotoxic medications, ultimately reducing the severity of AKI and its consequences. 
In summary, clinicians should be aware of the strengths and limitations of diagnostic tests ordered in AKI patients, as seen with the overly optimistic results in small, flawed UE and FENa studies. While we have taken a step in the right direction with diagnostic and prognostic biomarkers for $\mathrm{AKI}$, we must apply rigorous study design to diagnostic

\section{References}

1. Lusica M, Rondon-Berrios H, Feldman L. Urine eosinophils for acute interstitial nephritis. J Hosp Med. 2017;12(5):343-345.

2. Leaf DE, Srivastava A, Zeng X, et al. Excessive diagnostic testing in acute kidney injury. BMC Nephrol. 2016;17:9.

3. Pahwa AK, Sperati CJ. Urinary fractional excretion indices in the evaluation of acute kidney injury. J Hosp Med. 2016;11(1):77-80.

4. Perazella MA, Bomback AS. Urinary eosinophils in AIN: farewell to an old biomarker? Clin J Am Soc Nephrol. 2013;8(11):1841-1843.

5. Muriithi AK, Nasr SH, Leung N. Utility of urine eosinophils in the diagnosis of acute interstitial nephritis. Clin J Am Soc Nephrol. 2013;8(11):1857-1862.

6. Perazella MA, Coca SG. Traditional urinary biomarkers in the assessment of hospital-acquired AKI. Clin J Am Soc Nephrol. 2012;7(1):167-174. tests under evaluation before adopting them into clinical practice. Only then can we move the field forward and improve patient care.

Disclosure: Nothing to report.

7. Weisbord SD, Palevsky PM. Strategies for the prevention of contrast-induced acute kidney injury. Curr Opin Nephrol Hypertens. 2010;19(6):539-549.

8. Ioannidis JP, Panagiotou OA. Comparison of effect sizes associated with biomarkers reported in highly cited individual articles and in subsequent meta-analyses. JAMA. 2011;305(21):2200-2210.

9. Mishra J, Dent C, Tarabishi R, et al. Neutrophil gelatinase-associated lipocalin as a biomarker for acute renal injury after cardiac surgery. Lancet. 2005;365(9466):1231-1238.

10. Schaub JA, Parikh CR. Biomarkers of acute kidney injury and associations with short- and long-term outcomes. F1000Res. 2016;5(F1000 Faculty Rev.):986.

11. McMahon BA, Koyner JL. Risk stratification for acute kidney injury: Are biomarkers enough? Adv Chronic Kidney Dis. 2016;23(3):167-178. 